

\section{DISCLAIMER}

Portions of this document may be illegible electronic image products: Images are produced from the best available original document. 


\section{DISCLAIMER}

This report was prepared as an account of work sponsored by an agency of the United States Government. Neither the United States Government nor any agency thereof, nor any of their employees, make any warranty, express or implied, or assumes any legal liability or responsibility for the accuracy, completeness, or usefulness of any information, apparatus, product, or process disclosed, or represents that its use would not infringe privately owned rights. Reference herein to any specific commercial product, process, or service by trade name, trademark, manufacturer, or otherwise does not necessarily constitute or imply its endorsement, recommendation, or favoring by the United States Government or any agency thereof. The views and opinions of authors expressed herein do not necessarily state or reflect those of the United States Government or any agency thereof. 
Proceedings of Second Specialists' Meeting on

\title{
SHIELDING ASPECTS OF ACCELERATORS, TARGETS AND IRRADIATION FACILITIES
}

\author{
CERN \\ Geneva, Switzerland \\ 12-13 October 1995
}

organised by the

OECD Nuclear Energy Agency

the Shielding Working Group of the Reactor Physics Committee - Japan

the Radiation Shielding Information Center - U.S.A. 


\section{SATIF-2 \\ Shielding Aspects of Accelerators, Targets and Irradiation Facilities}

Particle accelerators have evolved over the last 50 years from simple devices to powerful machines, and will continue to have an important impact on research, technology and lifestyle. Today they cover a wide range of applications, from television and computer displays in households to the investigation of the origin and structure of matter. It has become common practice to use them for material science and medical applications.

In recent years, requirements from new technological and research applications have emerged, such as increased particle beams intensities, higher flexibility, etc., giving rise to new radiation shielding aspects and problems.

These proceedings review recent progress in radiation shielding of accelerator facilities, and evaluate advancements with respect to international co-operation in this field. 


\title{
THE PHOTONEUTRON YIELD PREDICTIONS BY PICA AND COMPARISON WITH THE MEASUREMENTS ${ }^{1}$
}

\author{
P. K. Job \\ Argonne National Laboratory, U.S.A. \\ T. A. Gabriel \\ Oak Ridge National Laboratory, U.S.A.
}

\section{Introduction}

The photoneutron yields at higher photon energies have become very important since the advent of high energy electron accelerators [1,2]. Bremsstrahlung is produced when the particle beam interacts with the storage-ring components or residual-gas molecules in the storage-ring vacuum. Bremsstrahlung thus produced interacts with the high- $Z$ materials in the beamline like the beam dumps and collimators to produce photoneutrons. There are three modes of neutron production by bremsstrahlung. At low energies $(<25 \mathrm{MeV})$, photons are absorbed by the dipole interaction and the compound nucleus thus formed decays emitting protons and neutrons and other heavier particles. At higher energies $(225 \mathrm{MeV})$, photon interacts with the nucleus through absorption on a quasi-deuteron, which subsequently decays producing a neutron and proton pair which can interact with the rest of the nucleus. At still higher energies the photopion production becomes possible and competes with the quasi-deuteron process. In this paper we have calculated the photoneutron yield from a thick copper target using the photonuclear interaction code PICA [3]. Using this as the neutron source, we have calculated the dose rates through heavy concrete and compared it with the measurements [4] made at the Advanced Photon Source at Argonne National Laboratory.

\section{PICA photonuclear interaction code}

PICA calculates the results of nuclear reaction caused by the collision of the photons with the nuclei [5]. PICA can do these calculations for incident monoenergetic photons as well as for bremsstrahlung spectra. For the dipole interaction the available cross-sections are used [6]. The higher energy interaction cross-sections are derived from the quasi-deuteron model of Levinger [7]. This is the photoabsorption by a neutron- proton pair. For photons above the pion threshold photon -pion production on nucleons is allowed to compete with the quasi-deuteron absorption process. The effect of the secondary nucleon- nucleus and pion-nucleus interactions following the photon absorption is calculated by the intranuclear cascade concept [8]. Each particle involved in the collision is traced through the nucleus using the appropriate particle-particle cross-sections until the particle escapes or is captured by the nucleus. In all parts of the calculation, the fermi momentum of the struck particle, the exclusion principle and the non-uniform density distribution of the nucleus are taken into account.

1 Work supported in part by U.S. Department of Energy, Contracts W-31-109-ENG-38 (Argonne National Laboratory), and DE-AC05-840R21400 (Oak Ridge National Laboratory). 
After the cascade process is complete, the nucleus is in an excited state and the excitation energy is dissipated through particle emission. The de-excitation of the nucleus is handled by the evaporation model [8].

\section{Geometry used for the simulation}

Figures 1 and 2 give the schematic and the simulated geometry for the PICA calculations. The particle beam during injection at the Advanced Photon Source can be partially or fully lost in one of the transition regions between the storage ring vacuum chamber and the insertion device straight section. The transition piece is a copper interface between the two vacuum chambers. The $56-\mathrm{cm}$ thick high density concrete ratchet wall is located $164 \mathrm{~cm}$ from the transition piece. The photon track lengths from the electromagnetic shower, when the injected particle beam is fully lost on the transition piece, were calculated by EGS4 [9]. The neutron yield from these photon track lengths was then calculated by PICA. These neutrons were transported by the one dimensional ANISN code using the $400 \mathrm{MeV}$ HILO86 cross-section library to estimate the photon and neutron dose rates outside the ratchet wall. The results are given in Table 1.

Table 1

Dose rates due to beam loss at the insertion device transition region during injection into the storage ring.

\begin{tabular}{|c|c|c|c|c|}
\hline & ChaRgE $^{a}$ & No. OF $\mathrm{e}^{-}$ & DOSE RATES & $(\mathbf{m r e m} / \mathbf{h})$ \\
\hline & / pulse & $/ \sec$ & NEUTRON & GAMMA \\
\hline Expt. 1 & $1.1 \mathrm{nC}$ & $6.87 \times 109$ & 26.4 & 1.5 \\
\hline Expt. 2 & $1.1 \mathrm{nC}$ & $6.87 \times 109$ & 39.6 & 1.1 \\
\hline PICA/ANISN & $1.1 \mathrm{nC}$ & $6.87 \times 109$ & 55.0 & 1.0 \\
\hline
\end{tabular}

a Injection rate is $1 \mathrm{~Hz}$.

\section{Measurements}

Radiation survey measurements were conducted outside the ratchet wall, while injected beam was being lost at one of the transition pieces. The primary objective of this was to study two potential beam loss scenarios, although some other measurements were also accomplished. First of all one of the corrector magnets adjacent to the transition piece was used at full strength to deflect the particle beam into the transition piece. Secondly, the beam was directed onto the closed gate valve which is just upstream of the transition piece. In both cases data was collected for 10 minutes while the charge entering the storage ring through the beamline transfer section was integrated. Prior to taking the data, the injection was tuned by the operating personnel to insure minimum loss between the booster transport system current monitor and the intended loss point.

The radiation survey instruments used for these measurements were a Victoreen 450P ionisation chamber for gamma radiation and an Eberline ASP-1 electronics package with a HP 2080 (Albatross) in the integral mode for neutrons. Prior to the commencement of the data taking, a survey determined the location of the maximum dose point along the length of the ratchet wall. Neutron monitors were 
placed at that location and at three other locations along the ratchet wall and a 5 minute count was taken in the integral mode. The gamma dose rate at the maximum dose location and the neutron dose rate at $90^{\circ}$ from the transition piece are given in Table I, along with the PICA predictions.

\section{Results and discussion}

The PICA simulation shows reasonable agreement with the measurements. The possible errors in this study are the beam loss scenario and the response of the neutron detectors to the high energy neutron radiation. The photoneutron yield from the transition piece depends on the shower development in the transition piece. Depending on the shower containment, this quantity and the resultant dose may vary at the most by a factor of three. For the present calculations we have assumed that only one-third of the electromagnetic shower is contained in the transition piece. The gamma dose rates outside the ratchet wall are negligible. This is attributed to the incomplete development of the electromagnetic shower in the copper transition piece (which is a maximum of $4 \mathrm{~cm}$ thick) so that much of the radiation is forward peaked and contributes to a resultant shower downstream. This also explains slightly elevated readings of the gamma dose rates at approximately $450 \mathrm{~cm}$ downstream of the transition piece on the ratchet wall. When incident on the ratchet wall, the shower encounters a much larger effective concrete thickness because of the shallow incidence angle. It can be seen from figure 1 that the slant thickness of concrete, downstream of the transition piece, is much large compared to the real thickness of $56 \mathrm{~cm}$. The increased gamma attenuation through the slant thickness of concrete accounts for low gamma radiation levels outside the ratchet wall.

\section{References}

[1] W. P. Swanson, "Radiological Safety Aspects of the Operation of Electron Linear Accelerators", IAEA Technical Series No. 188 (1979).

[2] H. J. Moe, "Advanced Photon Source: Radiological Design Considerations" ANL- APSLS-141 (1991).

[3] T. A. Gabriel et al., "PICA, An Intranuclear Cascade Calculation for High Energy Photon-Induced Nuclear Reactions", ORNL- 4687 (1971).

[4] P.K. Job and H. J. Moe, ANL- APS- LS 243 (1995).

[5] T. A. Gabriel and R. G. Alsmiller, "Photoneutron Disintegration at High Energies", ORNL-TM-2481 (1969).

[6] B. L. Berman, "Atlas of Photoneutron Cross Sections", UCRL 78482 (1976).

[7] J. S. Levinger, Phy. Rev., 84 (1951) 43.

[8] H. W. Bertini, "Intranuclear Cascade Calculations of the Secondary Nucleon Spectra from Nucleon-Nucleus Interactions", Phy. Rev. 188 (1969).

[9] W.R. Nelson et al., "EGS4 Code System", SLAC 265 (1985). 


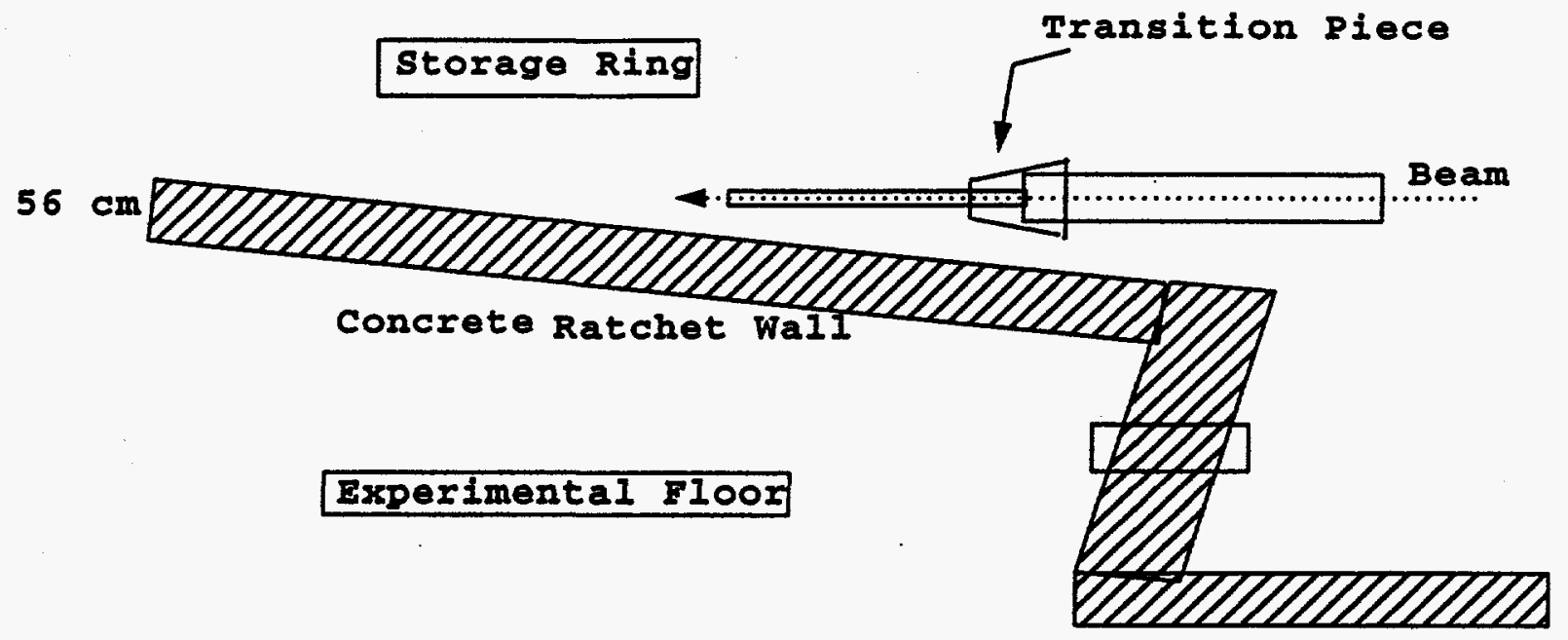

Figure 1 Beam loss configuration

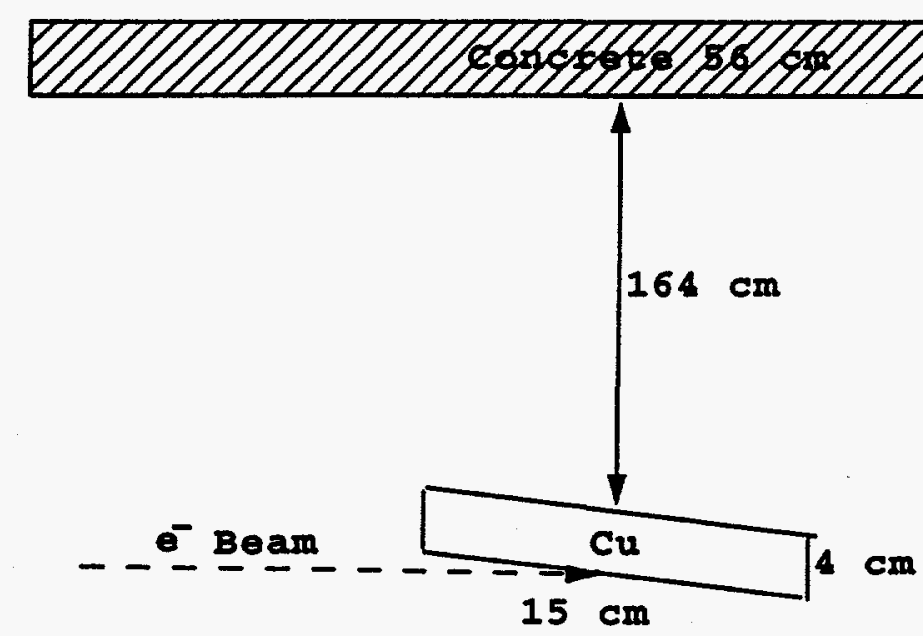

Figure 2 Geometry used for PICA simulation 